\title{
EDITORIAL
}

\section{Families and carers - their role}

\author{
David Skuse
}

Behavioural and Brain Sciences Unit, Institute of Child Health, London, UK, email d.skuse@ich. ucl.ac.uk
This issue's thematic papers consider the role played by families and carers in the management of major mental illness and dementia. Within many countries with well developed health services, the former asylum system of care has been replaced by 'care in the community', but asylums are still widely used in low- and middle-income parts of the world. The convention of psychiatric care by doctors, with the attendant medical model of mental illness, has, though, become less prominent in the past 50 years. Besides a widening range of experts providing services to those with mental illness and dementia, there is a trend towards engaging patients themselves in their management, as well as their families and other carers. These issues are discussed by Javed and Herrman in the context of developing and sustaining partnerships with patients and carers to deliver better mental health services in both high-income and middle- and lowincome countries. They point out that the World Health Organization supports this trend, as does the Royal College of Psychiatrists. There could be disadvantages though, and they acknowledge the possibility of there being an associated financial penalty to the families who are expected to provide that care.

In Arabic culture there is a long history, going back many centuries, of family obligation to sick relatives. Even when asylums were first established in the Arab world, in the 13th century, families were engaged in the care of people with mental illness, according to the article by Loza and Effat. This tradition began to change when
Western powers came to exert their influence and introduced a different style of care, based on an institutionalised health service. Stigma towards people with a mental illness allegedly increased, as the distance from their communities grew and their prominence in their own communities decreased. The authors emphasise that, although care in the community has undoubted benefits, it brings with it a greater burden on those tasked with providing care, especially if there are few professional supports in place because of financial constraints.

Finally, we segue into the report from Chaaya and colleagues concerning families in Lebanon who are looking after a relative with dementia. As the proportion of elderly members in our population grows, so the number of those with dementia increases disproportionately. In Lebanon, the burden of care largely falls upon middle-aged daughters, who are consequently often unable to work outside the home, unless they employ paid staff. The financial and emotional stress on such family carers is considerable, and was measured objectively in this investigation.

While the aim of engaging patients and their families in discussions about their care, and the form of that care in the community, is laudable and influential, this theme emphasises that this new model of care has had unforeseen consequences. The financial burden and stress of providing such care must be acknowledged by mental health professionals and policy makers wherever it is introduced.

\section{THEMATIC} PAPER

\section{Involving patients, carers and families: an international perspective on emerging priorities}

\author{
Afzal Javed ${ }^{1}$ and Helen Herrman ${ }^{2}$
}

'The Medical Centre, Manor Court Avenue, Nuneaton. UK email afzal.javed@ntlworld.com 2Orygen, The National Centre of Excellence in Youth Mental Health, and Centre for Youth Mental Health, University of Melbourne, Australia; President Elect, World Psychiatric

Association, email h.herrman@ Association, emain
unimelb.edu.au
This paper focuses on the importance of involving patients, families and other carers when offering and deciding on treatment and care options. It highlights the activities of international and national organisations in facilitating collaboration with patients and families in treatment, research, teaching and training related to mental healthcare.
The treatment and care of a person living with mental illness require a comprehensive and evidence-based approach that includes support for the active participation of that patient and their family. The relationship among different stakeholders in this equation - patients, families and practitioners - is, however, perceived differently by each. The patients and their families often experience limited opportunity to offer information 